\title{
CORPOREITÀ E IMMAGINARIO NEL DISCORSO MEDICO SETTECENTESCO: GLI ÉLÉMENTS DE PHYSIOLOGIE DI DIDEROT
}

\author{
GIANNI IOTTI (*)
}

SuNTO. - L'articolo parte da una serie di considerazioni sulla precocità degli interessi di Diderot in campo medico. «Non ci sono libri che io legga più volontieri dei libri di medicina, non ci sono uomini la cui conversazione sia più interessante, per me, di quella dei medici», egli arriverà ad affermare verso la fine della vita. Di fatto il discorso medico, nella sua opera, si identifica con un discorso filosofico militante all'insegna di un rigoroso monismo polemicamente opposto al dualismo della tradizione platonico-cristiana, un discorso nettamente orientato verso la totale traduzione dell" anima' nel funzionamento degli organi corporei. Ma al di là della polemica filosofica, in Diderot il sapere medico si rivela alla base d'una affascinante visione biologica. A differenza di un Buffon, il quale continua a riconoscere una centralità ontologica all'uomo, Diderot tende a negare ogni soluzione di continuità fra essere umano e regno animale - e addirittura tra regno animale e regno minerale. Di una simile posizione l'articolo mette in luce alcune conseguenze capitali sulle idee estetiche di Diderot; e, da qui, le riflessioni sul radicamento del pensiero nel corpo presenti negli Éléments de physiologie vengono ricondotte a uno dei più grandi cambiamenti di paradigma culturale della moderna storia europea.

$* * *$

RÉsumÉ. - L'article part d'une série de considérations sur la précocité des intérêts de Diderot dans le domaine médical. «Pas de livres que je lise plus volontiers que les livres de médecine, pas d'hommes dont la conversation soit plus intéressante pour moi que les médecins», il affirmera vers la fin de sa vie. En fait, le discours médical, dans son œuvre, s'identifie à un discours philosophique militant sous le signe d'un monisme rigoureux polémiquement opposé au dualisme de la tradition platonicienne-chrétienne, un discours nettement orienté vers la traduction totale de 'l'âme' dans le fonctionnement des organes du corps. Cependant, au-delà de la controverse philosophique, chez Diderot la connaissance médicale se révèle comme la base d'une fascinante vision biologique. Contrairement à un Buffon, qui continue de reconnaître une centralité ontologique à l'homme, Diderot tend à nier toute solution de continuité entre l'être humain et le règne animal - et même entre le règne animal et le règne minéral. L'article met en

(*) Università di Pisa, Italia. E-mail: gianni.iotti@unipi.it 
évidence quelques conséquences capitales d'une telle position sur les idées esthétiques de Diderot; et, d'ici, les réflexions sur l'enracinement de la pensée dans le corps présentes dans les Éléments de physiologie sont ramenées à l'un des plus grands changements de paradigme culturel de l'histoire européenne moderne.

\section{IL CORPO COME UBI CONSISTAM DEL SOGGETTO}

Vorrei cominciare con una citazione tratta dalla Lettre sur les aveugles:

... je n'ai jamais douté que l'état de nos organes et de nos sens n'ait beaucoup d'influence sur notre métaphysique et sur notre morale, et que nos idées les plus purement intellectuelles, si je peux parler ainsi, ne tiennent de fort près à la conformation de notre corps ${ }^{1}$.

Da questa posizione l'autore del Rêve de d'Alembert non si è mai dipartito, e la sua ultima opera, intitolata Éléments de physiologie in omaggio ad Albrecht von Haller (autore degli Elementa physiologiae corporis bumani, 1775), è un trattato che afferma ad ogni riga il primato assoluto del corpo sullo 'spirito'. Quello per la medicina, in fondo, non ha mai cessato di essere l'interesse principale di Diderot: «Pas de livres que je lise plus volontiers que les livres de médecine, pas d'hommes dont la conversation soit plus intéressante pour moi que les médecins», egli afferma (anche se la frase, va precisato, termina con una chiusa ironica: «mais c'est quand je me porte bien»)2. Tra il 1744 e il 1747 Diderot traduce, insieme ad altri, il Medicinal dictionary (1743-1745) di Robert James, pubblicato in francese con il titolo Dictionnaire universel de médecine (1746-1748), opera che rappresenta una tappa importante nel passaggio dal tardo iatromeccanicismo ancora dominante verso la metà del Setttecento al vitalismo che s'imporrà successivamente, proprio anche grazie a Diderot. Per lui si tratta prevalentemente di un impiego 'alimentare', ma il gergo medico e l'attitudine scientifica influenzeranno fortemente il filosofo e, in seguito, sarà soprattutto l'impegnativo ruolo

1 D. Diderot, Euvres philosophiques, Paris, 'Bibliothèque de la Pléiade' Gallimard, 2010, 139.

2 D. Diderot, Éléments de physiologie, édition critique, avec une introduction et des notes par J. Mayer, Paris, Librairie Marcel Didier, 1964, 299. 
di direttore dell'Encyclopédie ad allontanarlo dallo studio della medicina che l'aveva attratto fin da giovane. A partire dal 1769, una volta liberato dal quel compito, Diderot lavorerà agli Éléments de physiologie fino al 1780 e forse anche dopo, finché le condizioni di salute glielo permetteranno. Questo libro incompiuto va dunque considerato come il culmine di interessi che si erano manifestati precocemente, nonché come il testamento ideologico del suo autore.

In Diderot il discorso medico è un discorso filosofico militante all'insegna di un rigoroso monismo opposto polemicamente al dualismo della tradizione platonico-cristiana, un discorso nettamente orientato verso la totale traduzione della nozione di 'anima' nel funzionamento degli organi corporei. «La neurologie - ha scritto Jean Mayer - intéresse Diderot comme base de la psychologie et comme moyen de réfuter l'animisme. En revanche il n'a guère approfondi l'étude des muscles: il ignore, par exemple, la différence entre muscles striés et lisses»'. Nell'ultimo capitolo degli Eléments de physiologie, intitolato Maladies, si può leggere: «Toute sensation, toute affection étant corporelles, il s'ensuit qu'il y a une médecine physique également applicable au corps, et à l'âme» ${ }^{4}$. Diderot prosegue, però, dicendosi convinto dell'impraticabilità di una tale medicina poiché questa - egli osserva - richiederebbe una conoscenza perfetta del corpo umano ottenible, in prospettiva, solo attraverso la pratica diffusa dell'esame autoptico, pratica fortemente osteggiata all'epoca:

Comment perfectionner la médecine? en multipliant, en rendant générale l'ouverture des cadavres ... La conservation des hommes, et les progrès de l'art de guérir, sont des objets si importants que dans une société bien policée les prêtres ne devraient recevoir les cadavres que des mains de l'anatomiste, et qu'il devrait y avoir une loi qui défendit l'inhumation d'un corps sans ouverture. Quelle foule de connaissances n'acquerrait-on pas par ce moyen?

Simili affermazioni dovrebbero bastare a fissare le coordinate del discorso medico di Diderot: da un punto di vista teorico, l'obiettivo è quello di ritrovare il senso dell'esistenza umana sul piano strettamente biologico; e complementarmente, da un punto di vista politico, l'obiet-

3 J. Mayer, Diderot homme de science, Rennes, Imprimerie bretonne, 1959, 299.

4 D. Diderot, Éléments de physiologie, cit., 301.

5 D. Diderot, Éléments de physiologie, cit., 303. 
tivo è quello di negare ogni fondamento alla dottrina cristiana dell'anima relegando i preti, ipso facto, al ruolo di inutili e falsi parassiti sociali.

Ritrovare l'uomo spirituale in quello fisico (secondo un'espressione di Charles Mercier Dupaty), ricondurre le funzioni dell'anima al funzionamento del corpo, riportare - insomma - il trascendente al fisico o, più precisamente, al biologico: tale, si sa, è stato l'obiettivo del pensiero medico dei Lumi. Organi, fibre, ghiandole, nervi, processi metabolici e cognitivi pensati in termini che diventeranno ben presto neurofisiologici hanno tolto sempre più spazio all'anima. Nell'articolo 'Affection' dell'Encyclopédie, dovuto a Diderot, si trova la seguente affermazione:

Telle est notre construction qu'à l'occasion de cet état d'âme dans lequel elle ressent de l'amour ou de la haine, ou du goût ou de l'aversion, il se fait dans le corps des mouvements musculaires d'où, selon toute apparence, dépend l'intensité ou la rémisssion de ces sentiments. La joie n'est jamais sans une grande dilatation du cœur, le pouls s'élève, le cœur palpite, jusqu'à se faire sentir; la transpiration est si forte qu'elle peut être suivie de la défaillance et même de la mort. La colère suspend ou augmente tous les mouvements, surtout la circulation du sang; ce qui rend le corps chaud, rouge, tremblant, etc. ... Le méchanisme est rarement tel que la liberté de l'âme en soit suspendue à l'occasion des impressions. Mais on ne peut douter que cela arrive quelquefois: c'est dans le méchanisme du corps qu'il faut chercher la cause de la différence de sensibilité dans différents hommes, à l'occasion du même objet. Nous ressemblons en cela à des instruments de musique dont les cordes sont diversément tendues; les objets extérieurs font la fonction d'archets sur ces cordes, et nous rendons tous des sons plus ou moins aigus ${ }^{6}$.

Nel corso del Settecento, com'è noto, la nozione e l'immagine del corpo hanno subito un'evoluzione profonda: da 'macchina' meccanica conoscibile more geometrico secondo le leggi fisiche del movimento, com'era stato raffigurato in precedenza nell'ambito del pensiero cartesiano - si pensi alla metafora della pompa idraulica di Harvey o a quella pneumatica di Boyle ${ }^{7}$-, il corpo si è trasformato in organismo vitale, affermandosi quale ubi consistam del soggetto umano e diventando in tal modo - tra l'altro - base indispensabile nel processo di elaborazione

6 D. Diderot, Choix d'articles de L'Encyclopédie, Paris, Editions du C. T. H. S., 2001, 73-74.

7 Vedi P. Quintili, Introduction à D. Diderot, Éléments de physiologie, Paris, Champion, 2004, 11-101, qui 14. 
della nozione di individuo-cittadino di cui la Rivoluzione francese, verso la fine del secolo, avrebbe sancito i principi giuridici fondamentali.

Mentre la medicina - e penso soprattutto, per quanto riguarda Diderot, alla scuola di Montpellier, e più specialmente all'opera dal titolo Recherches anatomiques sur la position des glandes, et sur leur action (1751) del dottor Théophile de Bordeu (1722-1776), il quale diventerà uno dei personaggi del Rêve de d'Alembert - andava elaborando un'idea di corpo vivente come materia 'sensibile', nei campi della produzione romanzesca e teatrale, nell'arte in genere, si imponevano parallelamente forme di rappresentazione dell' homme sensible che modificavano radicalmente il senso stesso del processo della mimesi artistica. In tali contesti la caratterizzazione fisica, la gestualità, la forma del volto e le modalità espressive - concepite in maniera assai diversa dalle rigide catalogazioni di ascendenza antica - diventano chiavi interpretative essenziali dell'essere umano pensato nella realtà della sua esperienza contingente, e non più proiettato nelle trasfigurazioni sublimate e universali dell'estetica classica. Diderot, in particolare, manifesta un grande interesse per la fisiognomica, che in quel momento si sta profondamente rinnovando e, nelle sue opere narrative e drammatiche, il corpo diventa un protagonista assoluto.

Ecco, ad esempio, tratta dal racconto Ceci n'est pas un conte, la famosa descrizione della reazione della protagonista femminile allorché apprende da parte dell'amato la totale indifferenza, l'avversione, che costui ha sviluppato nei suoi confronti:

À ces mots une pâleur mortelle se répandit sur son visage; ses lèvres se décolorèrent; les gouttes d'une sueur froide qui se formaient sur ses joues, se mêlaient aux larmes qui descendaient de ses yeux; ils étaient fermés; sa tête se renversa sur le dos de son fauteuil; ses dents se serrèrent; tous ses membres tressaillaient; à ce tressaillement succéda une défaillance qui me parut l'accomplissement de l'espérance qu'elle avait conçue à la porte de cette maison. La durée de cet état acheva de m'effrayer. Je lui otai son mantelet, je desserrai les cordons de sa robe, je relâchai ceux de ses jupons, et je lui jetai quelques gouttes d'eau fraîche sur le visage. Ses yeux se rouvrirent à demi, il se fit entendre un murmure sourd dans sa gorge; elle voulait prononcer, Je lui suis odieuse, et elle n'articulait que les dernières syllabes du dernier mot. Puis elle poussait un cri aigu, ses paupières s'abaissaient, et l'évanouissement reprenait ${ }^{8}$.

8 Vedi D. Diderot, Contes et romans, Paris, 'Bibliothèque de la Pléiade' Gallimard, 2004, 510. 
Il narratore, come si vede, enumera gli effetti fisici di uno stato d'animo con la precisione degna di un rapporto medico. Il focus della narrazione si è concentrato sulle reazioni del corpo del personaggio. Il personaggio stesso, potremmo dire, è concepito prioritariamente come un corpo che reagisce in un certo modo a certe sollecitazioni. Ed è, questo, un fenomeno generale nella letteratura europea tra Sette e Ottocento, con ricadute di fondamentale importanza nell'economia della narrazione. Ora, è interessante notare come, passando dal discorso di finzione al discorso propriamente medico, Diderot non faccia che riprodurre un analogo interesse per le relazioni che, nel soggetto umano, s'instaurano tra un dato psicologico e le sue manifestazioni corporee.

Così, negli Éléments de physiologie, l'evocazione del genio artistico all'opera (si tratta di uno scultore) può servire a mostrare il modo in cui le passioni si riproducono in movimenti 'mimetici' del corpo secondo un modello propriamente 'patognomico' di descrizione del corpo per usare il termine di Buffon - rapportato alla dimensione temporale:

... ses yeux sont ardents, ses mouvements sont prompts et troublés; il halète, la sueur lui coule du front, il contrefait du visage la passion qu'il veut rendre: ... Si c'est la colère, il grince les dents; si c'est la tendresse, il s'abandonne; si c'est le désespoir, ses traits s'allongent, sa bouche s'entrouvre, ses membres se raidessent; si c'est le mépris, sa lèvre supérieure se relève; si c'est l'ironie, il sourit malignement; je lui tâte le pouls, il a la fièvre?.

Un simile passo documenta bene l'avvento della nuova estetica anti-classica, che i Romantici svilupperanno, secondo la quale l'opera d'arte è una vera e propria appendice della soggettività, anche corporea, dell'artista. Ma a noi, qui, interessa sottolineare l'assenza di soluzioni di continuità che Diderot postula tra il sentimento interiore e la sua estrinsecazione fisica. Oggetto di semiosi o soggetto di emozioni, nelle riflessioni mediche e nelle rappresentazioni artistiche del Settecento il corpo vivente si sottrae alla topica codificata dei repertori fisiognomici tradizionali, e rinvia a una fenomenologia peculiare ormai assai lontana dal rigido e immaginifico meccanicismo barocco del secolo precedente.

9 D. Diderot, Éléments de physiologie, cit., 52. 


\section{UN'AFFASCINANTE PROSPETTIVA BIOLOGICA}

L'approccio di Diderot alla medicina si inscrive in una prospettiva 'psico-somatica' che, del resto, si ritrova già parzialmente nel pensiero medico antico ${ }^{10}$. Il che spiega, tra l'altro, osservazioni problematiche come questa: «L'amour est plus difficile à expliquer que la faim: car le fruit n'éprouve pas le désir d'être mangé» ${ }^{11}$. Per altro, la posizione di Diderot è radicalmente anti-antropocentrica. A differenza di un Buffon il quale, per non discostarsi totalmente dall'idea della presenza di un piano divino soggiacente alla creazione, riconosce all'uomo una superiorità e una centralità ontologiche all'interno del sistema biologico, Diderot tende a negare ogni soluzione di continuità fra essere umano e regno animale - e addirittura, nel Rêve de d'Alembert se non negli Éléments de physiologie, tra regno animale e regno minerale. Per lui le più alte facoltà intellettuali dell'uomo, che il persistente antropocentrismo a sfondo religioso dell'epoca identificava con una scintilla divina, sono solo una modalità dell'istinto animale che si è evoluto nel tempo; una capacità 'perfettibile e perfezionata' ${ }^{12}$ sempre compromessa da stati nervosi, menomazioni, malattie, sostanze tossiche, sollecitazioni contingenti. La statica descrizione degli organi attrae meno Diderot di quanto non faccia lo studio del loro comportamento, in cui egli ritrova le pre-

10 Nel Timeo, ad esempio, Platone - dopo la famosa metafora spaziale che dispone le tre specie di anima in rapporto ai vari organi del corpo - riporta i disordini dell'anima a due specie: la pazzia e l'ignoranza. Ma tali disordini hanno tutti una causa fisica (o culturale dovuta all'educazione), al punto che è possibile assimilare la posizione di Platone a una sorta di determinismo biologico e culturale: « ... perché nessuno è volontariamente malvagio, ma per non so qual cattivo abito del corpo e per difetto d'educazione il malvagio diventa malvagio; e questo è un malanno per ognuno e ad ognuno capita suo malgrado. D'altra parte, anche quanto ai dolori, l'anima soffre il maggior male per via del corpo; giacché, quando gli umori delle pituite acide e salse e tutti gli altri amari biliosi, vagando per il corpo, non trovino respiro al di fuori, ma, avvolgendosi internamente, si confondano dopo di aver commista la propria evaporazione col moto dell'anima, producono malattie psichiche d'ogni sorta, ora più ora men gravi, ora più ora meno frequenti ... » (Platone, Tutte le opere, Firenze, Sansoni, 1983, 1128-1129, 1139).

11 D. Diderot, Éléments de physiologie, cit., 277.

12 «L'homme est aussi une espèce animale; sa raison n'est qu'un instinct perfectible et perfectionné; et dans la carrière des sciences et des arts il y a autant d'instincts divers que de chiens dans un équipage de chasse»: D. Diderot, Réfutation suivie de l'ouvrage d'Helvétius intitulé L'Homme, in Euvres philosophiques, cit., 518. 
tese proprietà dell' anima' tradotte in termini fisiologici. Ecco, a proposito del senso della vista, una descrizione dell'occhio in rapporto a determinate emozioni: «L'œil s'obscurcit dans la peur et dans la tristesse, s'allume dans la colère, brille dans l'amour. Dans l'amour il est humide, sec dans la colère, et quelquefois sanglant» ${ }^{13}$.

Più precisamente, Diderot osserva come sugli organi possano agire stimolazioni sia morali che fisiche, e sottolinea come le prime non siano affatto meno potenti delle seconde: «La peur fait cesser le hoquet; - scrive - un récit produit le dégoût, même le vomissement. Toutes les sortes de désir agissent sur les glandes salivaires, mais surtout le désir voluptueux» ${ }^{14}$. Ma è sulla reciprocità delle relazioni tra dimensione morale $\mathrm{e}$ dimensione fisica che egli insiste, allo scopo di rigettare la prospettiva dualista e giungere a una definizione organica della materia vivente:

Effet réciproque de la sensation sur les objets, et des objets sur la sensation. Je suis heureux, tout ce qui m'entoure s'embellit. Je souffre, tout ce qui m'entoure s'obscurcit. Mais ce phénomène n'a lieu que dans les plaisirs, ou dans les peines modérées. Un peu de bile dont la circulation dans le foie est embarassée change toute la couleur des idées; elles deviennent noires, mélancoliques, on se déplaît par tout où l'on est ... Et c'est à de pareilles causes que tient notre raison, nos goûts, nos aversions nos désirs, notre caractère, nos actions, notre morale, nos vices, nos vertus, notre bonheur, et notre malheur, le bonheur et le malheur de ceux qui nous entourent ${ }^{15}$.

Un corollario essenziale della tendenza di Diderot a pensare gli esseri viventi in termini organici è quello della instabilità e della labilità dei caratteri delle varie specie: «L'ordre général de la nature change sans cesse: - si legge negli Éléments de physiologie - au milieu de cette vicissitude la durée de l'espèce peut-elle rester la même? Non: il n'y a que la molécule qui demerure éternelle, et inaltérable» ${ }^{16}$. Così il discorso medico non può che aprirsi alle vertiginose prospettive biologiche del Rêve de d'Alembert, evocate in quell'opera tramite l'artificio letterario delle visioni di un sognatore e assunte qui, invece, come considerazioni positive dello scienziato. La serie di variazioni senza soluzione di

13 D. Diderot, Éléments de physiologie, cit., 224.

14 D. Diderot, Éléments de physiologie, cit., 25.

15 D. Diderot, Éléments de physiologie, cit., 231.

16 D. Diderot, Éléments de physiologie, cit., 42. 
continuità che, in uno spaccato sincronico, Diderot postula dalla pietra all'uomo, dalla materia inorganica all'organismo biologico più evoluto, viene proiettata in diacronia nel sistema di un perpetuo cambiamento degli esseri che contiene elementi della teoria trasformista, presenti anche in Buffon, i quali passando da Lamarck e Geoffroy de SaintHilaire arriveranno fino a Darwin. Così, ad esempio, a proposito del problema della generazione, su cui si affrontavano varie scuole con teorie spesso fantasiose, Diderot resta prudente, ma la sua insistenza nel livellare le specie, con lo scopo di umiliare l'orgoglio antropocentrico, resta una costante: «Je serais tenté de ramener la génération de l'homme à celle du polipe qui se reproduit par division» ${ }^{17}$; oppure: «Ce pépin n'a qu'un développement nécessaire. C'est un arbre; ainsi de l'homme» ${ }^{18}$, si legge sempre negli Éléments de physiologie. Va tenuto conto, tuttavia, che il 'trasformismo' virtuale di Diderot si inscrive ancora nella visione linearista della 'catena degli esseri' di ascendenza idealistico-platonica che si ritrova anche in Linneo, visione influenzata dalle antiche idee sul rapporto analogico tra macrocosmo e microcosmo a cui Leibniz aveva dato una formulazione moderna con la sua teoria dell'armonia prestabilita. Perché si affermi il modello diacronico ramificato dell'essere vivente in continua evoluzione, infatti, bisognerà aspettare la Philosophie zoologique (1809) di Lamarck ${ }^{19}$.

La lezione di fisiologia di Diderot va concepita come una lezione volta non solo a liberare la natura umana da qualunque ipoteca metafisica, ma anche a togliere all'essere umano ogni illusione sull'unicità e sull'immutabilità della propria identità biologica, e a proporre un'idea di materia 'sensibile' tendenzialmente indifferenziata i cui caratteri si riproducono da specie a specie in una serie infinita di modulazioni ${ }^{20}$.

17 D. Diderot, Éléments de physiologie, cit., 189.

18 D. Diderot, Éléments de physiologie, cit., 194.

19 In compenso, soprattutto nelle sue ultime opere come gli Éléments o la Réfutation d'Helvétius (ma anche nel Rêve de d'Alembert), il suo vitalismo porta Diderot a non cadere nella visione semplicistica dello iatro-meccanicismo.

20 A causa del suo materialismo vitalistico, che tende a stemperare la specificità del corpo all'interno dell'universale catena dell'essere, Diderot finisce per ridurre il soggetto umano a un insieme di sensazioni, chimismi, funzioni percettive e facoltà cognitive, azioni e reazioni riflesse che rischia di lasciare fuori quella complessa nozione di io-coscienza che negli stessi anni, da un punto di vista diverso, andava elaborando Rousseau. E ciò anche se egli, riprendendo nozioni sensiste, ha cura di distinguere tra 
Ora, va notato che un simile tentativo di razionalizzazione dei fenomeni vitali in senso materialistico può condurre Diderot a prendere sul serio leggende o miti. Così, ad esempio, la sua concezione radicalmente funzionale del sentimento materno riportato alla sensazione piacevole procurata da una sollecitazione d'ordine corporale sperimentata dalla femmina che allatta, lo porta ad evocare forme di allattamento assai improbabili, col risultato paradossale di reintrodurre - surrettiziamente - l'elemento irrazionale in un discorso che si vorrebbe scientifico. Si consideri ad esempio la notazione seguente:

Toutes les mères femmes ou animales ne nourrissent qu'à la condition d'y trouver du plaisir; le nourrisson chatouille la nourrice par le téton: de là la tendresse des nourrices pour des enfants qui les tétent bien. C'est une longue manière de jouir ... exemples de la prédilection des mères pour les enfants qui tétent bien, c'est-à-dire chatouillent bien, c'est la préférence que les vaches donnent aux serpents: une fois tétées par ces serpents, elle ne souffrent plus la main. Le toucher est plus fort que la vue: ce qui explique le cas des chiennes qui allaitent des chats. ${ }^{21}$

Un'analoga considerazione potrebbe valere per il tema della 'mostruosità', ricorrente negli Éléments de physiologie (tema assai diffuso, del resto, nel dibattito medico-filosofico dell'epoca). Così come le turbe, le malattie, i traumi - secondo Diderot - sono alterazioni del funzionamento degli organi che compromettono la combinazione di molecole a cui è riducibile il singolo essere, e quindi la sua struttura biologica, così l'esistenza dei mostri - vale a dire di individui che presentano particolari differenze di conformazione esterna del corpo o interna degli organi rispetto ai loro simili - prova che i tratti ritenuti propri di una specie o di un genere sono di fatto una convenzione e che la materia vivente possiede infinità di combinazioni in cui si ritrova una perenne unità e complementarità di caratteri (perciò l'uomo - dice Diderot - è il mostro della donna e viceversa). Così, quelle che Voltaire aveva chiamato le 'singolarità della natura', riferendosi implicitamente a una norma naturale insieme infranta e confermata da fenomeni eccezionali,

una conscience de l'existence e una conscience de soi: la prima legata alla pura sensazione di esistere nell'attimo, tipica delle forme di vita inferiori; la seconda, caratteristica dell'uomo, fondata in ogni soggetto dalla memoria della propria esistenza e in grado di formare il sentimento dell'identità personale.

21 D. Diderot, Éléments de physiologie, cit., 134-135. 
diventano per Diderot la dimostrazione dell'impossibilità stessa di fissare una norma:

Tous les êtres circulent les uns dans les autres, par conséquent toutes les espèces ... tout est un flux perpétuel ... Tout animal est plus ou moins homme; tout minéral est plus ou moins plante; toute plante est plus ou moins animal. Il n'y a rien de précis en nature ...22

egli afferma nel Rêve de d'Alembert. In Diderot, è stato scritto, lo studio dei mostri:

... nuance la conception de l'être vivant, en écarte tout finalisme et détruit toute idée d'harmonie préétablie. Les monstres jouaient dans la Lettre sur les aveugles ... le rôle d'êtres transitoires entre le chaos et notre univers relativement équilibré: les voici devenus une nécessité statistique ... Dans les Éléments de physiologie ils représenteront la règle universelle ${ }^{23}$.

In effetti, al di là del mostro che viene considerato tale a causa della sua anomalia in rapporto alla specie a cui appartiene, Diderot arriva a concepire la specie stessa, in chiave trasformistica, come un 'mostro plurale', transitorio, la cui mostruosità si palesa agli occhi dello scienziato a un grado di astrazione superiore. Così egli può chiedersi:

Pourquoi l'homme, pourquoi tous les animaux ne seraient-ils pas des espèces de monstres un peu plus durables? Pourquoi la nature qui extermine l'individu en peu d'années, n'exterminerait-elle pas l'espèce dans une longue succession de temps? L'univers ne semble quelquefois qu'un assemblage d'êtres monstrueux. Qu'est-ce qu'un monstre? Un être dont la durée est incompatible avec l'ordre subsistant ${ }^{24}$.

\section{FISIOLOGIA ED ESTETICA}

Detto questo, non bisogna mai dimenticare che il Diderot scienziato è inscindibile dal Diderot filosofo, artista e teorico dell'estetica. Se, facendo dell'universo biologico una realtà piena di 'mostri', egli

22 D. Diderot, Le Rêve de d'Alembert, in Euvres philosophiques cit., 311.

23 J. Mayer, Diderot homme de science, cit., 242.

24 D. Diderot, Éléments de physiologie, cit., 208-209. 
intende prima di tutto contestare la teoria del fissismo creazionistico e il preteso ordine immutabile dell'universo, nello stesso tempo questa sua posizione lascia trasparire l'interesse per una mostruosità intesa in accezione diversa, una mostruosità irreale fatta di desideri immaginari e di fantasie, di un 'punto di vista' irriducibile alla ragione convenzionale. Vale a dire l'interesse per ciò che possiamo chiamare l'irrazionale', per quei contenuti legati alla logica del sogno, del fantastico, della follia che, in campo artistico, erano stati dominanti in ambito barocco e che, in modi diversi, lo saranno di nuovo più che mai in ambito romantico e post-romantico. È coerente, in tal senso, che la struttura dei nervi e gli effetti nervosi siano al centro del discorso medico di Diderot. Si legge negli Éléments de physiologie:

L'action des nerfs porte au cerveau des désirs singuliers, les fantaisies les plus bizarres, des affections, des frayeurs: il me semble que j'entends crier ma femme; on attaque ma fille, elle m'appelle à son secours; je vois les murs s'ébranler autour de moi; le plafond est prêt à tomber sur ma tête; je me sens pusillanime, je me tâte le pouls, j'y découvre un petit mouvement fébrile: la cause de ma frayeur connue, elle cesse ${ }^{25}$.

Edgar Allan Poe o Kafka meglio di altri, si potrebbe osservare, sapranno sfruttare stati nervosi di questo tipo per creare straordinari incubi letterari - privi ormai, però, del rassicurante ritorno all'ordine razionale che troviamo in Diderot.

Un tale accostamento del pensiero medico-scientifico del philosophe settecentesco ai maestri ottocenteschi o novecenteschi del fantastico letterario non dovrebbe sorprendere più di tanto, se è vero com'è vero che si deve proprio alla ragione illuministica matura la postulazione, su basi nuove, di una dimensione dell'esperienza psichica (quella del sentimento, della sensibilità, dell'emozione) che si sottrae alla nozione tradizionale di razionalità. Il Settecento, ha affermato lo storico americano Peter Gay, è meno il secolo del trionfo della Ragione che quello della sua crisi e della rivolta contro il Razionalismo ${ }^{26}$. A questo proposito va sotto-

25 D. Diderot, Éléments de physiologie, cit., 92.

26 «The philosophes'glorification of criticism and their qualified repudiation of metaphysics make it obvious that the Enlightenment was not an Age of reason but a Revolt against Rationalism. ... The claim for the omnicompetence of criticism was in no way a claim for the omnipotence of reason. It was a political demand for the right to 
linato il contributo che Diderot ha dato indirettamente alla scoperta di ciò che, da Freud in poi, si chiamerà l'inconscio'. Come non definire decisamente 'freudiano', in effetti, il seguente, straordinario, passo:

Je suis porté à croire que tout ce que nous avons vu, connu, entendu, aperçu, jusqu'aux arbres d'une longue forêt, que dis-je, jusqu'à la disposition des branches, à la forme des feuilles, et à la variété des couleurs, des verts et des lumières; jusqu'à l'aspect des grains de sable du rivage de la mer, aux inégalités de la surface des flots agités par un souffle léger, soit écumeux et soulevés par les vents de la tempête, jusqu'à la multitude des voix humaines, des cris des animaux et de bruits physiques, à la melodie et à l'harmonie de tous les airs, de toutes les pièces de musique, de tous les concerts que nous avons entendus, tout cela existe en nous à notre insu ${ }^{27}$.

Partendo dall'assolutizzazione della sensazione e dagli effetti delle sollecitazioni a cui gli organi sono sottoposti, Diderot perviene all'idea moderna - proustiana, verrebbe da dire - di memoria affettiva e, quindi, a quella di pensiero inconscio, alimentato dalle innumerevoli vestigia mnestiche della nostra vita interiore, così come la psicanalisi l'ha definito. Idea che si ritrova anche, ma in modi diversi, nell'invenzione rousseauiana dell'io autobiografico tramite l'evocazione di ricordi che sprofondano nell'esperienza sensoriale. Si legge, in proposito, nelle Confessions di Rousseau:

Durant six mois entiers, je ne sortis pas une seule fois que pour aller chez maman ou à l'Église, et je n'en fus pas même tenté. Cet intervalle est un de ceux où j'ai vécu dans le plus grand calme, et que je me suis rappelés avec le plus de plasir. Dans les situations diverses où je me suis trouvé, quelques-uns ont été marqués par un tel sentiment de bien-être qu'en les rémemorant j'en suis affecté comme si j'y étais encore. Non seulement je me rappelle les temps, les lieux, les personnes, mais tous les objets environnants, la température de l'air, son odeur, sa couleur, une certaine impression locale qui ne s'est fait sentir que là, et dont le souvenir vif m'y transporte de nouveau ${ }^{28}$.

question everything, rather than the assertion that all could be known or mastered by rationality»: P. Gay, The Enlightenment: An interpretation. 1. The rise of modern paganism, New York/London, W. W. Norton \& Co., 1995, 141.

27 D. Diderot, Éléments de physiologie, cit., 241.

28 J.-J. Rousseau, Les Confessions, livre III, in CEuvres complètes, Paris, 'Bibliothèque de la Pléiade' Gallimard, 1959, vol. I, 122. 
Attraverso l'apertura di così vasti spazi dell'immaginazione e della memoria oltre i limiti della coscienza, Diderot non fa che perseguire il suo disegno anti-antropocentrico, erodendo quel potenziale di autodeterminazione che l'io classico era disposto a spartire - per così dire solo con Dio. E lo fa concependo il soggetto umano non tanto come animale agente in base ai riflessi di una volontà univoca, quanto come 'insieme' di animali - gli organi - ognuno dotato di capacità sensitive e di una propria 'volontà' relativamente autonoma: «L'homme peut donc être regardé comme un assemblage d'animaux où chacun garde sa fonction particulière, et simpathise soit naturellement soit par habitude avec les autres $\rangle^{29}$. È evidente come una metafora del genere riduca la 'volontà' dell'individuo a una mera sommatoria di risposte a stimoli provenienti dai vari organi che formano il suo corpo: «La volonté est l'effet d'une cause qui la meut et la détermine; un acte de volonté sans cause est une chimère. Rien ne se fait par saut dans la nature. Tout y est lié. L'animal, l'homme, tout être est soumis à cette loi générale» ${ }^{30}$. E ancora: «L'on dit que le désir naît de la volonté. C'est le contraire: C'est du désir que nâit la volonté. Le désir est fils de l'organisation, le bonheur et le malheur fils du bien-être, ou du mal-être. On veut être heureux $\rangle^{31}$. Il grande problema filosofico della libertà può così venire tradotto in termini brutalmente fisiologici:

Examinez-ce qui se passe en vous, ce n'est jamais vous qui voulez manger ou vomir, c'est l'estomac; pisser, c'est la vessie; et ainsi des autres fonctions. Veuillez tant qu'il vous plaira, il ne s'opérera rien, si l'organe ne le veut aussi. Vous voulez jouir de la femme que vous aimez, mais quand jouirez-vous? Quand l'organe le voudra ${ }^{32}$.

L'immagine dell'uomo che Diderot ci consegna nella sua operatestamento, dunque, è quella di un soggetto i cui moventi sono sottoposti a una sorta di fatalismo biologico, la cui libertà si riduce a una serie di azioni-reazioni di ordine chimico e nervoso che avvengono nel suo corpo, la cui facoltà cognitiva razionale è continuamente soggetta a interferenze da parte di altre forme di attività psichica.

29 D. Diderot, Éléments de physiologie, cit., 287.

30 D. Diderot, Éléments de physiologie, cit., 262.

31 D. Diderot, Éléments de physiologie, cit., 265.

32 D. Diderot, Éléments de physiologie, cit., 287-288. 
Una tale visione materialistica dell'essere vivente - come si diceva sopra - lascia inevitabilmente fuori quella nozione di io-coscienza che negli stessi anni, da un punto di vista diverso, Rousseau andava elaborando. Si assiste così, negli Éléments de physiologie, a una atomizzazione 'neurofisiologica' dei centri di pensiero che si spargono per tutto il corpo e, complementarmente, a una 'migrazione' dell'io dalle regioni astratte della pura razionalità a quelle biologiche costituite dai meccanismi fisiologici degli organi. Ma ciò che in una simile traslazione va perduto in termini di capacità auto-riflessiva della coscienza viene in parte recuperato - se così ci si può esprimere - attraverso l'individuazione del ruolo essenziale di facoltà 'creative' come la memoria e l'immaginazione:

L'imagination ressuscite dans l'homme les voix, les sons, tous les accidents de la nature, les images qui deviennent autant d'occasions de s'égarer. L'homme à imagination se promène dans sa tête comme un curieux dans un palais, où ses pas sont à chaque instant détournés par des objets intéressants. Il va, il revient, il n'en sort pas. L'imagination est l'image de l'enfance que tout attire sans règle ${ }^{33}$.

Al di qua della sua connotazione parzialmente negativa, è inutile sottolineare la portata, in particolare per quanto riguarda gli sviluppi futuri nei campi dell'estetica e dell'espressione artistica, di un simile nesso tra immaginazione e pensiero infantile: di lì a pochi decenni, per Chateaubriand il genio non sarà che memoria, per Baudelaire la metafora nascerà dalla combinazione sinestetica di antiche tracce sensoriali, e Rimbaud proclamerà che je est un autre esaltando una 'alterità' che, in fondo, procede dalla vita del corpo e dall'immediatezza delle sensazioni. Con ciò, naturalmente, non si vogliono certo negare i limiti della fisiologia razionalistica di Diderot - né da un punto di vista filosofico o psicologico né, tanto meno, da un punto di vista strettamente medico. Resta che i risultati a cui l'autore degli Éléments de physiologie giunge, nelle sue riflessioni sul radicamento del pensiero nel corpo, gli assegnano un posto importante all'interno di uno dei più grandi cambiamenti di paradigma culturale della storia del pensiero europeo.

33 D. Diderot, Éléments de physiologie, cit., 250. 
